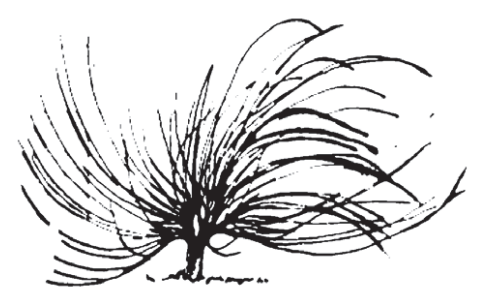

\title{
Aspectos Sociales, Políticos y Filosóficos de la Educación de Niños Talentosos
}

\author{
Shirley Segura Chanto ${ }^{1}$ \\ Universidad Técnica Nacional \\ Alajuela, Costa Rica \\ shisegura@hotmail.com
}

"El talento no se basa en tener dones que los demás no, sino en saber utilizarlos"

\begin{abstract}
Resumen
El talento es un tema que muchos creen conocer y saber tratar o estimular, pero la realidad es que muy pocas personas pueden identificar el verdadero talento. En Costa Rica, los niños talentosos pasan desapercibidos, en ocasiones por sus padres y hasta por los docentes, con muy poca ayuda para su identificación y un tratamiento que no cumple con sus necesidades básicas. Ellos tienen los mismos derechos que los demás y merecen recibir una educación de acuerdo con sus necesidades educativas en donde se estimulen los talentos que poseen, sin ser confundidos con niños aplicados o con facilidad de aprendizaje. A nivel educativo, los niños talentosos no cuentan con muchas herramientas, ya que los docentes no poseen la capacitación necesaria para identificarlos y brindarles la estimulación en el talento en que sobresalen, además de la necesidad de adecuar los programas para sus necesidades. Lastimosamente, y se describirá más adelante, esta situación es constante y está presente en las aulas del
\end{abstract}

Recibido: 9 de setiembre de 2013 - Aprobado: 12 de febrero de 2014

1 Curriculista, Universidad Técnica Nacional. Máster en Currículum y Docencia Universitaria, ULACIT y bachiller en Enseñanza del Inglés, Universidad Nacional 
país. A continuación, el ensayo describe tres perspectivas en torno a este tema, en donde se ampliarán las ideas anteriormente expuestas.

Palabras clave: niños talentosos, perspectivas sociales, políticas y filosóficas, niños brillantes

\begin{abstract}
Talent is a topic that most people think they know, stimulate and deal with, but the truth is that few people can identify real talent. In Cost Rica, there are talented children that are not noticed, sometimes by their own parents and even by teachers, with little help to identify it and a treatment that does not fulfill their basic needs. They have the same rights and deserve an education according to their needs, in which their talents can be stimulated without their being confused with gifted children. At school, children do not have enough tools because teachers do not have the knowledge or training to identify them and provide the stimulation for their talent for them not only to highlight but also to adjust the programs to their needs. This is unfortunate, and it will be described later that this is a constant situation in this country. In the following essay, three perspectives around this topic will be described, and the ideas mentioned before will be elaborated on.
\end{abstract}

Keywords: talented children, social, political and philosophical perspectives, gifted children

\title{
Perspectiva Social
}

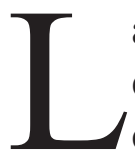

a educación de los niños talentosos es un tema que se puede escuchar frecuentemente entre los docentes y psicopedagogos cuando se analizan diagnósticos de este tipo. Varios de ellos, han logrado investigar generalidades de los niños talentosos pero aún sigue débil en la parte de capacitación a padres, educadores y médicos, quienes están al cuidado del niño. En ocasiones, la falta de conocimiento del tema y la confusión que pueden tener las personas con los niños aplicados o con facilidades para aprender, causa errores que no permiten 
el desarrollo normal en la vida de estas personas. A partir de aquí, se abarcará el enfoque social desde el punto de vista de educadores y familiares para poder comprender los conceptos, tratamiento y técnicas para mejorar el estilo de vida de estos niños.

Para empezar, es importante conocer la diferencia entre los términos que se utilizan para definir esta situación en los niños. Munro (2002), explica lo que es considerado como gifted children o niño talentoso, que son quienes poseen una motivación innata y el deseo imparable de aprender múltiples temas y poder responder los numerosos cuestionamientos que poseen. En español, existe otro término que puede confundirse con el anteriormente mencionado: niños brillantes. Estos tienen la habilidad para desarrollar de manera excelente tareas explícitamente enseñadas, ya sea a nivel cognitivo o físico, como lo es el área del deporte o de las artes. Mientras, el término gifted children en inglés, se refiere a los niños que presentan habilidades que no han sido explícitamente desarrolladas en el proceso de enseñanza - aprendizaje formal.

Para ser más específico, el término de niños talentosos es definido más claramente por Munro (2002), como niños con altas competencias intelectuales, quienes son creativos y socioemocionales, son personas que no han sido capacitadas para ciertas actividades, pero que sobresalen naturalmente en estas por cuestiones genéticas. Además, son sobresalientes en más de una tarea que realizan. En la parte social, son un poco suprimidos e introvertidos por las diferencias que pueden tener con respecto al resto de sus compañeros, ya que, al ser personas con intereses diferentes, tienen dificultades para socializar y compartir con los demás.

Por otra parte, "The Council of Curriculum, Examinations and Assessment" (2006), brinda un reporte acerca de lo que los niños talentosos y brillantes deben tener tanto a nivel familiar como escolar. Es importante tomar en cuenta que las actividades de las que pueden disfrutar los niños talentosos son bastante diferentes a las de quienes no lo son. Como por ejemplo, Porquicho (2012), dice que los niños talentosos disfrutan de campamentos en donde logran compartir con personas iguales, y se sienten comprendidos y acompañados, además de que tienen la posibilidad de hacer amigos, que es una de las consecuencias que sufren a raíz del talento, pues como menciona también "The Council of Curriculum, Examinations and Assessment" (2006), ellos pueden también presentar algún tipo de problema de aprendizaje como 
la dislexia, el Síndrome de Asperguer o dificultad motora y sensorial. Esta situación, los limita a compartir con compañeros que no poseen las mismas afinidades y gustos.

Ante estas situaciones, es necesario tomar en cuenta los aspectos intrafamiliares y personales que pueden enfrentar los niños, como lo son la frustración y el fracaso académico expresadas por Bernal (1997), quien asimismo, menciona el interés que posee en enseñar a las personas diferentes destrezas físicas, psicomotoras y de estudio, pero sin perder la parte socio - interpersonal.

\section{Perspectiva Política}

Desde la perspectiva política de la educación de los niños talentosos, es necesario apuntar a varias direcciones, entre las cuales están las políticas instituidas en otros países, y la manera en como está establecido en Costa Rica. Además, es necesario enfocarse en las que han tenido éxito en el lugar en donde se han implementado para encontrar la clave de tal avance. Para empezar a describirlas, Saleiro (1995), explica la importancia de la atención de los niños talentosos en Portugal, específicamente en la parte de la integración a la sociedad.

Saleiro (1995), hace referencia a que antes de la publicación de la Ley de Necesidades Educativas Especiales en ese país en 1991, se tomaban en cuenta solamente los niños con trastornos físicos, emocionales o conductas marginales. Estos eran, para muchas personas, los que realmente necesitaban de esas reformas. Después de este cambio, se han podido integrar a estos niños en clases extra escolares, en donde se les estimula un poco más a desarrollar los talentos que poseen. Esto ha logrado que estos niños se puedan integrar exitosamente a la sociedad.

Otro ejemplo al que se puede recurrir es el caso de Argentina. En este país, fue a través de la Ley Federal de la Educación, promulgada en 1993, en la que se incluyeron los niños talentosos. Vergara (2006), menciona que a partir de esta Ley han surgido departamentos especializados para salvaguardar el bienestar de estas personas, para que sean integrales en todo sentido. Además, esta Ley apunta a la educación para la diversidad, atendiendo a cada ser humano en sus necesidades educativas particulares, y se tiende a tomar el término educación de lo heterogéneo con el respeto que se merece cualquier ser humano. Incluso, menciona que ya existen sanciones si se violentan los derechos que 
poseen las niñas y los niños talentosos, lo cual marca un gran avance en el país en este aspecto.

Algo más que se puede observar en Argentina y que tiene mucha relevancia, es el hecho de que ejemplifica cada paso a seguir con los niños talentosos: desde la detección temprana por medio de estrategias especializadas, la integración y participación de estos niños en el ámbito educativo, social y laboral, el mejoramiento de la calidad de la oferta pedagógica para educar adecuadamente a sus alumnos, hasta propiciar la participación activa de los padres, quienes deben compartir el compromiso en el proyecto educativo de los hijos. Esta es una visión integradora, donde estos niños puedan contar con una enseñanza personalizada en el aula común, tomando en cuenta el contexto social del aula y equilibrando las necesidades por medio de adaptaciones curriculares. En Argentina, estas adaptaciones tienen el apoyo de un equipo de profesionales en la educación especial, es decir, cuentan con el soporte requerido para el éxito en la planificación educativa y aplicación de la ley, pues pueden adaptar un currículum individual para la ampliación y profundización de los contenidos del currículo (Vergara, 2006).

Otra nación que se enfoca en el bienestar de los niños talentosos es España. Este país posee también una serie de lineamientos. Por ejemplo, Torrego et al. (2011), explican la importancia de las clases especiales y escuelas especializadas, con modelos curriculares aptos y un alto porcentaje de trabajo colaborativo para que los niños puedan agruparse con sus pares y con las demás personas con el fin de lograr más éxito en la parte social. Además de estas especificaciones, se mencionan las adaptaciones que se le pueden hacer al currículum para explorar todas sus capacidades. Este ejemplo demuestra que realmente son países preocupados por el desarrollo completo e integral del ser humano, en donde se reconocen las diferentes necesidades de cada uno y se les ayuda a sobrellevar de la mejor manera todos sus requerimientos brindando la ayuda requerida.

A pesar de estas particularidades tan importantes en estos países, es necesario hacer referencia a las divergencias que estos presentan. Mientras que en Argentina hay integración en el aula con una adecuación curricular, en España hay segregación e institucionalización especializada. Esto demuestra que las perspectivas que se presentan en cada país en que se piensa ayudar a estas personas son variadas, pero siempre en vista a mejorar su estilo de vida y aprendizaje. 
Ahora toca ver lo que sucede en Costa Rica. En este país ya existe la Ley para la Promoción de la Alta Dotación, Talentos y Creatividad en el Sistema Educativo Costarricense (2009), esta es la ley número 8899, que se promulgó en 2009 y fue publicada en el año 2010. Esta Ley supone una serie de cambios a seguir para el desarrollo de este tipo de personas, entre las cuales se mencionan la capacitación y creación de reglamentos referentes a la Ley. Morales (2012), comenta que dos años después de la publicación de la Ley, aún no existe un reglamento, pero que se va a empezar a realizar. Por su parte, insta al Ministerio de Educación Pública (MEP) a ponerse a trabajar en esta reglamentación, a brindar la capacitación necesaria y establecer convenios con universidades que puedan brindar la atención debida a las niñas y niños talentosos.

Por otro lado, Venegas (2012), expresa la necesidad de trabajar fuerte en esta área, pues la cantidad de niños talentosos podría ser mayor que la que se cree existe, ya que en ocasiones se realizan exámenes de aptitud a niños y niñas de preescolar para identificarlos, aunque estas pruebas no son del todo relevantes, pues no evalúan cualitativamente, por lo cual, no son una prueba totalmente válida para la detección en esa edad en la que sería indispensable realizarlo.

Las pruebas que se realizan en preescolar son meramente cuantitativas, pues se debe rendir un examen en el que con cierta cantidad de actividades se evalúan aspectos específicos del desempeño de los niños que al final suman cierta cantidad de números y brinda una nota final. Ellos deben realizar ejercicios para conocer la edad maduracional, como lo son dibujos o formas. Además, se realizan ejercicios físicos de atrapar la pelota con una y otra mano, y actividades kinestésicas específicas que tienen cierto puntaje a la hora de ser valoradas y dar el veredicto final del examen, este punto fue concedido por C. Villalobos (comunicación personal, 24 de agosto de 2012).

Por otra parte, la observación cualitativa puede descifrar aspectos específicos de conducta que puedan referir más específicamente a características propias de los niños talentosos, pues son situaciones más conductuales que requieren más de un día de observación. Además, estas pruebas deberían ser aplicadas por profesionales en la rama para poder identificarlos correctamente, situación que no se da en la actualidad. Por las razones descritas anteriormente, es necesario diseñar un examen específico en el que se pueda identificar a un niño talentoso en 
diversos momentos de su vida, no solamente con una prueba que limite sus conocimientos o talentos a los descritos en una prueba.

Incluso, el Consejo Superior de Educación (CSE) (2009), en una de sus actas, menciona que desea realizar un análisis de la política en Educación Especial, en donde desean incluir a los niños talentosos. Este acuerdo debe ser tomado en cuenta por la Ley. Este importante detalle es indolente y va en contra de lo que dice el Código de la Niñez y la Adolescencia. A pesar de que se menciona lo que sucede y la importancia de la necesidad de realizar una reforma, no se toma ningún acuerdo, más que solamente la mención al tópico de interés.

Además de lo anterior, Monturiol (2005), comparte la necesidad de trabajar fuertemente en ésta área, pues se ha podido corroborar que la población de estos niños podría ser mayor a la que se cree que hay actualmente. Por ejemplo, Castro, Pereira, Solís, Ureña y Villalobos (2010), demuestran por medio de un estudio realizado en Heredia, el trato que reciben los niños talentosos de varias escuelas de la provincia. Se pudo corroborar la falta de políticas educativas adecuadas, pues no se han podido lograr ni cumplir con lo que los niños talentosos merecen, como por ejemplo, una adecuación curricular para la estimulación de sus talentos e intereses, así como la comprensión e integración en la sociedad con las demás personas, tomando en cuenta que sus intereses son diferentes a los de los demás de su misma edad. Incluso, toman en consideración la opinión de algunos niños que manifiestan el deseo de tener clases con actividades más creativas y retadoras.

Un aspecto interesante que surge a partir de esta investigación, es que el MEP se encuentra ausente en directrices para el trabajo con esta población y no existe un apoyo curricular. Solamente, algunos docentes hacen referencia a la importancia de completar los temarios, para lo cual no cuentan con el tiempo suficiente, ni la guía para desarrollar otros temas de interés con estos niños.

Otro ejemplo, que se puede utilizar, también descrito por Castro, Pereira, Solís, Ureña y Villalobos (2010), es que los docentes de escuelas tanto públicas como privadas en donde realizaron el estudio, desconocen que exista una Ley relacionada con esto, y la identificación de estos niños es nula, ya que en ocasiones están con cantidades de entre 26 y 40 niños en el aula, con los cuales es difícil identificar alguna necesidad especial. Además, no cuentan con la capacitación o instrumentos para 
identificación; sin embargo, se vuelve a mencionar la preocupación de los docentes de terminar los temarios del MEP.

Finalmente, al analizar estas pruebas referentes a las políticas educativas de estos países que se han creado con el fin de brindar ayuda, las niñas y los niños talentosos, se puede observar de primera entrada, que las políticas educativas de Costa Rica, en este aspecto están bastante atrasadas con respecto a otros países, pues por ejemplo en Argentina, la Ley se contempló desde el año 1993 (Vergara, 2007), mientras que en Costa Rica, se decretó hasta el año 2010, y aún hoy en día, no se cuenta con el reglamento para la correcta aplicación de la Ley en beneficio de niños que están a la espera de mejores oportunidades. El MEP no se ha pronunciado aún con alguna propuesta para este reglamento, ni mucho menos con los profesionales que se necesitan para poder atender a estos niños, tanto a nivel preescolar como escolar, y mucho menos con respecto a la capacitación que necesitan los docentes. Asimismo, el MEP, no menciona ninguna flexibilización curricular para poder aplicar la Ley tal y como se propone, ni algún plan de acción para considerarlo. Lastimosamente se puede comprobar que, es difícil ir más allá de lo que se escribe en el papel, pues se han podido observar muchos planes o ideas, pero es un hecho que no se ha tomado ninguna acción al respecto.

\section{Perspectivas Filosóficas}

Dentro del marco de las perspectivas filosóficas en el tema de los niños talentosos, se debe tomar en cuenta las razones por las cuales se deben enseñar. Para iniciar, el Código de la Niñez y Adolescencia (1998), trata múltiples temas relacionados con los derechos de los niños, niñas y adolescentes, pero el capítulo en el que se enfocará esta perspectiva es en el quinto, que es el que habla acerca de la educación. El caso de los niños talentosos está explícito en el artículo 56, en donde se menciona que "Las personas menores de edad tendrán el derecho de recibir educación orientada hacia el desarrollo de sus potencialidades" (p. 13). Este artículo se puede interpretar de varias maneras, pero en este caso se deberían respetar las necesidades educativas especiales para desarrollar un currículum especial.

Además de lo que se menciona en el artículo 56, el artículo 58, inciso a) dice: "Garantizar educación de calidad e igualdad de oportunidades para las personas menores de edad" (p. 13). También, el artículo 
62, que se lee de la siguiente manera: "Las personas con un potencial intelectual superior al normal o con algún grado de discapacidad, tendrán el derecho de recibir atención especial en los centros educativos, para adecuar los métodos de enseñanza a sus necesidades particulares" (p. 14), $y$ hace mención a los niños talentosos, es acá en donde se especifica que tendrán derecho a recibir atención especial en los centros educativos y que se deben adecuar los métodos de enseñanza a sus necesidades específicas. Todos estos artículos señalan lo que deben recibir, como norma, y que deberían tener en una sociedad como esta.

Por esta razón, se debe tomar en cuenta que desde esta perspectiva, hay que responder la pregunta ¿Para qué educar a los niños talentosos? Se podría responder, con la razón de que simplemente ellos son personas con los mismos derechos y deberes que cualquier otro niño o adolescente merece en la sociedad, por lo que todos los aspectos curriculares deben adecuarse a ellos también.

Otro punto importante, para tomar en cuenta es que la educación es un derecho de todos por igual, en tanto, además de ser gratuita sin exclusión y bajo los mismos requisitos para todos. En la Declaración Universal de los Derechos Humanos (1948), se menciona que la educación tiene como objeto el pleno desarrollo de la personalidad humana y las libertades fundamentales. En relación con el tema en cuestión, es importante enfocarse en que se deben dar situaciones óptimas para el desarrollo de la personalidad, pues se presentan situaciones que son excluyentes para esta población. Como ya se conoce, uno de los problemas esenciales que se deben tomar en cuenta es la dificultad en la socialización de estos niños, se deben adecuar los métodos para facilitar su aprendizaje y la potenciación de sus talentos.

Por esta razón, es importante notar que en muchos sentidos se señala el bienestar de estas personas, no solo físico sino a nivel social también. En ocasiones las personas pueden adaptarse al sistema, pero en otras es necesario adaptar el sistema a estas personas para que esto se dé realmente. Está dicho y hasta decretado y se debe velar por que se cumpla lo que todas las personas sin distinción alguna deban recibir. Como ejemplo a esto, López (2009), describe en el artículo las características de los niños talentosos y sus cualidades excepcionales de la siguiente manera: 
Son niños que requieren programas y/o servicios educativos superiores a los que de manera habitual proporciona el programa escolar (...) Los niños capaces de altas realizaciones incluyen aquellos que han demostrado su alto rendimiento y/o potencialidad en cualquiera de las siguientes áreas, por separado o en combinación. (párr. 9).

De acuerdo con el autor, estas personas son identificadas de alto rendimiento y requieren programas o servicio educativos superiores a los que normalmente se usan, para poder contribuir con: "la capacidad intelectual general, aptitudes académicas o específicas, pensamiento productivo o creativo, capacidad de liderazgo, capacidad en artes visuales o representativas y capacidad psicomotriz"(párr. 9). Igualmente, el autor citado, rescata que las personas talentosas no son homogéneas, pues existen factores biológicos, psicológicos, socioeconómicos y culturales entre otros, diferentes como cualquier grupo de seres humanos. Además, brinda características específicas de estas personas, como su alto rendimiento académico en todas las asignaturas, la disciplina y responsabilidad, pero muy especialmente que son excepcionalmente inteligentes, autosuficientes y problemáticos, solitarios, introvertidos y que no necesitan ayuda, pues su talento les basta, por lo que les cuesta socializar en especial con las personas de su edad. Entonces, ¿Está el currículo adaptado a personas con estas características?

Además de comprender las características del niño talentoso, es importante saber cómo se puede detectar en el aula y de qué manera se le puede adaptar la clase a sus necesidades. Por ejemplo, Otero (2009), especifica que

el docente debe atender a las calificaciones del niño, a los distintos informes que posea, a los resultados obtenidos en las evaluaciones iniciales al principio del curso, analizar los trabajos de clase, observar el comportamiento en clase así como obtener información de la familia y del comportamiento del niño en casa.

Además, menciona que se debe hacer pruebas subjetivas para evaluar la conducta para conocer datos sobre la inteligencia, motivación o interés. Pero, ¿Estarán los docentes preparados para identificarlos? ¿Se cumplen los ideales de cada estudiante? 
De acuerdo con lo anterior, se puede suponer que estos niños que tienen necesidades educativas especiales, tienen los mismos derechos que las demás personas, por lo que se les debe brindar la misma calidad de educación que ellos merecen, especialmente el dejar de ser confundidos y más bien integrados a la sociedad, pero muy especialmente identificados desde la edad preescolar, con el fin de que el desarrollo de ellos sea mejor año con año y su talento bien estimulado.

Además, la Pontificia Universidad Católica de Valparaíso (2008), en un documento en que se describe un programa especial para las personas talentosas, determina claramente rasgos importantes e indispensables a conocer acerca de los niños talentosos, por ejemplo, rasgos de personalidad, creatividad, comportamiento social, motivación, la parte emocional y aptitudes relacionadas con el diario vivir de estas personas. Explica acerca de un programa que se desarrolla en esa Universidad, con la cual se trabaja con niños talentosos con un currículum desarrollado para explorar las aptitudes y retarles a un aprendizaje más exigente. Es interesante que se mencione que estos proyectos, pueden tanto atraer o no al estudiante talentoso, pues ellos poseen intereses y características diferentes, lo que hace aún más difícil identificarlos. Se manifiesta, además que el talento no puede observarse directamente, que este se infiere a partir de ciertas características o conductas de los estudiantes, ya que es una cuestión genética que se da sin ninguna distinción.

Con todo lo anterior, se puede resumir que en general todas las personas tienen derechos, y en la Declaración Universal de los Derechos Humanos está planteado, en cuanto a brindar situaciones optimas para el desarrollo de la personalidad y de las capacidades de todos los seres humanos, no se puede omitir el hecho de que las personas talentosas existen, que se les debe mostrar la misma atención que a las que no lo son, que las cosas por las que sobresalen pueden ser igualmente apreciadas y atendidas, además de estimuladas. Hay que dejar de lado que las personas que necesitan ayuda curricular son solamente las que presentan algún tipo de dificultad para el aprendizaje. Las personas talentosas tienen todo el derecho de recibir la ayuda que merecen, pero más que todo la guía para que se puedan desarrollar como las demás personas. 


\section{Conclusiones}

Finalmente, se cree que el talento es un tema que muchos creen conocer y saber tratar o estimular, pero la realidad es que muy pocas personas pueden identificarlo. Se pudo comprobar la necesidad de restablecer el currículo existente y en la elaboración y seguimiento de un reglamento que permita hacer cumplir los derechos universales que cada individuo posee.

Estas personas viven el día a día sin seguimiento alguno, más que con el apoyo de sus allegados y quienes los logran comprender. De la misma forma en que en algún momento defendieron las personas con dificultades de aprendizaje y se les logró modificar el currículum de acuerdo con las necesidades que presentaban, es necesario poder hacerlo en este país con las personas talentosas. Esto se puede realizar, tomando como ejemplo países que desde hace más de 10 años pudieron avanzar en este aspecto y lograron adaptar los programas para estas personas, Costa Rica, debe apresurar su paso en el seguimiento a la Ley y la aprobación del respectivo reglamento. No se puede concebir que a casi dos años de su promulgación, estas personas sigan esperando conocer qué va a ser de ellos.

Actualmente, en Costa Rica no se cuenta con elementos o instrumentos para identificar esta situación. No se tiene conocimiento acerca de capacitaciones a personal docente para poner en práctica la Ley y todo lo que propone, siendo ellos mismos quienes tienen mayor contacto con estas personas y quienes deberían poder observar y analizar sus conductas. No se puede saber cuántas personas mal identificadas se encuentran en este momento intentando averiguar cómo mejorar las distintas áreas de "deficiencia" que puedan mostrar.

Es necesario actuar, tan pronto como sea posible, para que estas personas que se encuentran en las aulas de instituciones tanto privadas como públicas puedan surgir y desarrollarse como cualquier otra. Se deben realizar actividades en que estas personas puedan socializar y tener contacto con otras como ellos, poder ser comprendidos y lograr enfocarse en la vida que cualquier persona merece, contar con un currículum diseñado para el desarrollo de talentos y la supervisión del personal idóneo y capacitado para este fin, en suma, ser tratado bajo la igualdad de oportunidades que todas las personas merecen. 


\section{Referencias bibliográficas}

Asamblea Legislativa de la República de Costa Rica. (1998). Código de la niñez y la adolescencia. Recuperado de http://cpj.go.cr/docs/derechos/codigo-ninez.pdf

Asamblea Legislativa de Costa Rica. (2009). Ley para la promoción de la alta dotación, talentos y creatividad en el sistema educativo costarricense. Recuperado de http://documentos.cgr.go.cr/content/dav/jaguar/USI/normativa/2009/Proyecto/

Bernal, E. (1997). Perspectivas multinacionales de la superdotación y la educación de los niños superdotados: La trilogía española [Versión Digital]. Revista Ideacción, (10) Recuperado de http://www.centrohuertadelrey.com/files/fck//file/ num_18 prespectivas_multinacionales.pdf

Castro, D., Pereira, Z., Solís, J., Ureña, K. y Villalobos, B. (2010). El talento en niños y niñas de I ciclo de la educación general básica: La perspectiva docente vrs la realidad educativa. Revista Educare, Vol. XIV, pp 97 - 112. Recuperado de http://www.revistas.una.ac.cr/index.php/EDUCARE/article/view/1530/1450

Consejo Superior de Educación. (2009). Acta Ordinaria No. 09 - 2009. Recuperado de http://www.cse.go.cr/pdf/ACTA09-23-09.pdf

Declaración Universal de los Derechos Humanos. (1948). Recuperado de http:// www.derechoshumanos.net/normativa/normas/1948-DeclaracionUniversal. htm?gclid=CNePir6OyLECFQW0nQodJRkA4Q

López, J (2009). Talentos, prodigios o genios. Efdeportes Revista Digital 137. Recuperado de http://www.efdeportes.com/efd137/talentos-prodigios-o-genios.htm

Monturiol, S. (2005). Escuela desatiende a infancia talentosa. Recuperado de http:// www.una.ac.cr/campus/ediciones/2005/marzo/2005marzo_pag03.html

Morales, K. (30 de junio de 2012) Mejores oportunidades para niños superdotados. La Nación. Recuperado de http://www.nacion.com/2011-02-07/Opinion/Foro/ Opinion2675280.aspx

Munro, J. (2002). Understanding and identifying gifted learning: Disabled students. Recuperado de https://students.education.unimelb.edu.au/selage/pub/readings/ giftedld/GLLDR-Understandidenti.pdf

Otero, N. (2009). ¿Qué hacer con un alumno de altas capacidades? Recuperado de http://www.eumed.net/rev/ced/09/nom5.htm

Pontificia Universidad Católica de Valparaíso. (2008). Programa Buenos Estudiantes con Talento Académico BETA - PUCV. Recuperado de_http://www.programabeta.cl/descargas/Guia_para_la_identificacion_de_alumnos_con_talentos_academicos.pdf

Porquicho, J. (27 de mayo de 2012). El peaje que hay que pagar por tener una mente privilegiada. Diario de Cádiz. Recuperado de: http://www.diariodecadiz.es/article/cadiz/1013560/peaje/pagar/por/tener/una/mente/privilegiada.html

Saleiro, M. (1995). Nuevas perspectivas, nuevas direcciones en el diagnóstico y desarrollo de los niños superdotados y talentosos en Portugal. Revista Ideacción, (6), 10 - 13. Recuperado de http://www.centrohuertadelrey.com/files/fck//file/ num_18_nuevas_prespectivas.pdf

The Council of Curriculum, Examinations and Assessment (2006). Gifted and talented children in (and out of) the classroom. Recuperado de http://www.edfac.unimelb.edu.au/eldi/selage/documents/GLT-Defininggiftedness.pdf 
Torrego, J. et al (2011). Alumnos con altas capacidades y aprendizaje colaborativo. Madrid, España: Fundación SM.

Venegas, E. (2 de diciembre del 2012). Niños, niñas y jóvenes talentosos en Costa Rica. La Extra. Recuperado de http://www.diarioextra.com/2010/diciembre/02/ opinion02.php

Vergara, M. (2006). Niños talentosos y marco legal en nuestro país. Revista E - Eccleston (5). Recuperado de http://ieseccleston.buenosaires.edu.ar/RevistaN\%BA5_Vergara_Panzeri.pdf

Vergara, M (2007). El proceso de Inclusión escolar de alumnos con necesidades educativas especiales: De la teoría a la práctica. Recuperado de http://www.cedalp. com/articulo34.htm 